\title{
NSS + 20 : un collectif interdisciplinaire en mutation ?
}

S'interroger sur le sens de l'interdisciplinarité vingt ans après la création de l'association NSS-Dialogues et de la revue Natures Sciences Sociétés : tel était l'enjeu du colloque qui s'est tenu à Cerisy-la-Salle du 30 septembre au 5 octobre $2013^{1}$. Ce colloque anniversaire a été l'occasion de mesurer le chemin parcouru. Il a aussi été l'occasion, pour le collectif NSS, de se confronter à d'autres approches interdisciplinaires et à de nouvelles thématiques de recherche afin de réaffirmer son identité.

L'identité est bien évidemment liée au contexte. À cet égard, celui du début des années 1990 était fort différent du contexte actuel, tant sur le plan de la politique internationale (les promesses du sommet de la Terre de Rio) que du paysage scientifique français où l'interdisciplinarité apparaissait comme marginalisée. Entre-temps, la crise écologique s'est approfondie et le changement climatique comme l'extinction massive des espèces sont désormais reconnus comme des faits avérés, sans que les politiques ayant succédé aux espoirs de Rio aient pris la mesure des transformations radicales à réaliser. La politique nationale en matière d'enseignement supérieur et de recherche a connu des bouleversements profonds, notamment au niveau de l'évaluation. Quant au statut des recherches interdisciplinaires, elles se trouvent prises dans une injonction contradictoire : l'interdisciplinarité apparaît de plus en plus comme un prérequis dans la plupart des appels à propositions de recherches tandis que les disciplines académiques semblent plus que jamais enfermées dans un mode d'évaluation standardisé qui ne laisse qu'une place très limitée aux travaux conduits en interdisciplinarité.

Comment tenir compte à la fois de l'héritage de vingt années d'une activité éditoriale exigeante, de qualité, se

\footnotetext{
1 Colloque « Interdisciplinarités entre Natures et Sociétés », sous la direction de Bernard Hubert et Nicole Mathieu, Cerisyla-Salle, Centre culturel international de Cerisy, du 30 septembre au 5 octobre 2013, http://www.ccic-cerisy.asso.fr/ interdisciplinaires13.html. Voir aussi, à la fin de ce numéro dans les pages de l'association NSS-Dialogues, le compte rendu de la journée de réflexion de l'association (6 décembre 2012) destinée à préparer le colloque de Cerisy.
}

voulant le reflet des préoccupations de son temps, et se projeter dans un futur, imprévisible par définition? L'ouverture a été la piste choisie par le comité d'organisation afin de répondre à ce double enjeu. Ouverture à l'international tout d'abord, avec des conférences de plusieurs chercheurs étrangers, représentatifs de courants de pensée jugés proches des préoccupations de NSS ; ouverture thématique ensuite, tant par la présence de chercheurs ne travaillant pas sur l'interface naturesociété que par l'organisation d'ateliers autour de thèmes proposés par les participants au colloque; ouverture générationnelle enfin, avec l'invitation faite à une quinzaine de jeunes chercheurs ayant déjà une expérience de l'interdisciplinarité.

On a pu constater que le projet initial de NSS reste pertinent : on ne peut traiter des questions environnementales que de manière interdisciplinaire et en lien fort avec la société. Mais l'actualité et l'urgence de ces questions, tout autant que les tensions entre disciplines, amènent à poser différemment la question de l'interdisciplinarité. Celle-ci n'apparaît plus tant comme un défi ni comme une fin en soi - si elle l'a jamais été. Tenue pour acquise par un certain nombre de participants au colloque, surtout parmi les plus jeunes, l'interdisciplinarité doit plus que jamais être une entreprise collective dont les enjeux ont surtout paru, au cours de ce colloque, se cristalliser autour de trois tensions fondamentales : l'objet et la fonction des collectifs interdisciplinaires, leur source de légitimation et leur engagement dans les débats de société.

Le statut des objets dans les recherches interdisciplinaires a toujours été source de débat à NSS - d'aucuns regrettant que, souvent, l'interdisciplinarité apparaisse comme une fin en soi, détachée de ses objectifs (la compréhension d'un problème complexe) quand d'autres refusent de ne constituer que des collectifs interdisciplinaires ad hoc. NSS a toujours cherché à articuler une conception de l'interdisciplinarité appliquée à des objets empiriques et une conception plus théorisée de la démarche. Mais où situer le curseur entre une interdisciplinarité pour soi et une interdisciplinarité « orientéeobjet»? À quel moment les chemins pris par les 
chercheurs engagés dans chacune de ces options divergent-ils ? Où sont leurs points de (re)convergences ? La question a été posée de manière originale par certains intervenants du colloque de Cerisy, et, en particulier, par les jeunes chercheurs. Ces derniers ont réalisé leur socialisation scientifique dans l'interdisciplinarité : celle-ci s'impose dans leurs démarches individuelles comme un fait évident qu'il ne faut surtout pas tenir pour acquis, mais sur lequel ils s'appuient pour poser différemment la question des collectifs interdisciplinaires. Pour eux, ces collectifs ne sauraient être fondés sur le rapprochement de différentes compétences disciplinaires orientées vers la résolution d'un problème, ni dans l'interdisciplinarité pour soi, mais sur la mise en commun de pratiques interdisciplinaires organisées autour de questions de société, voire de postures scientifiques. Comment faire exister ces collectifs dans leur unicité et leur diversité ?

La question de la légitimation académique de ces collectifs, en particulier dans leurs rapports aux disciplines, a bien entendu été posée. La légitimation d'une démarche interdisciplinaire est-elle à rechercher dans les disciplines d'origine des chercheurs, dans un assemblage de disciplines en perpétuelle reconstruction, ou bien encore dans des communautés épistémiques qui seraient organisées autour de démarches, de questions de société et/ou d'objets de recherche? Le point précédent nous a montré que la dernière piste gagne aujourd'hui du terrain... Si NSS est resté à l'affût de ces mutations au cours de ces dernières années en faisant une place à certaines communautés épistémiques émergentes comme la political ecology, l'Alliance pour la résilience ou encore la sustainability science, la prise en compte des enseignements de Cerisy doit nous inviter à être encore plus attentifs à l'actualité de ces courants et à prendre au sérieux les questions que l'engouement qu'ils suscitent pose pour l'interdisciplinarité. Quelles innovations les collectifs interdisciplinaires rendent-ils possibles ou impossibles ?

L'engagement des chercheurs, et, plus généralement, le rapport entre science et action, sont apparus comme un troisième thème récurrent. Pour de nombreux participants à Cerisy, cet engagement ne mérite pas de longs débats ; il s'impose plutôt comme une évidence. Pourtant les formes que revêtent les collaborations entre les chercheurs et la société, entre les différentes conceptions des savoirs, leurs hybridations, génèrent encore bien des questions, comme en témoigne par exemple l'éditorial du premier numéro de 2013 (NSS, 21, 1). De surcroît, l'évidence mentionnée ci-dessus reste à légitimer dans les pratiques de recherche et l'évaluation. Les institutions sont-elles prêtes à reconnaître aux chercheurs la part de subjectivité inhérente à tout engagement ? Certaines des communautés épistémiques interdisciplinaires que nous avons évoquées assument clairement cette position, sans que la légitimité de leur démarche soit remise en question dans l'évaluation scientifique. Où puisent-elles cette légitimité ?

En somme, c'est la question de la construction, de l'articulation et de la légitimation de collectifs interdisciplinaires fondés sur un continuum entre connaissance et action qui a été (re)posée à Cerisy. Le colloque anniversaire a clairement fait apparaître que NSS avait un rôle majeur à jouer par rapport à ces collectifs. Partageant une culture et une histoire communes, les membres de NSS assument un certain nombre de valeurs, d'engagements et de pratiques scientifiques ; c'est aussi un lieu de débats où différentes options quant aux formes et aux fonctions de l'interdisciplinarité ont toujours su coexister. Il reste que le colloque de Cerisy a mis en évidence que ce positionnement et ce rôle doivent être plus clairement affichés, en particulier au regard des trois points évoqués ci-dessus.
Xavier Arnauld de Sartre, Olivier Petit 